\title{
CORRIGENDUM
}

\section{Cnn1 inhibits the interactions between the KMN complexes of the yeast kinetochore}

Lucy J. Bock, Cinzia Pagliuca, Norihiko Kobayashi, Ryan A. Grove, Yusuke Oku, Kriti Shrestha, Claudio Alfieri1, Cristina Golfieri, Amanda Oldani, Marianna Dal Maschio, Rodrigo Bermejo, Tony R. Hazbun, Tomoyuki U. Tanaka and Peter De Wulf

Nat. Cell Biol. 14, 614-624 (2012); published online 6th May 2012; corrected online 8th February 2013

In the version of this Article that was originally published, the acknowledgement to M. Winey was omitted in error. The first sentence of the Acknowledgements section should read:

"The authors thank A. Amon (MIT, USA), S. Biggins (University of Washington, USA), D. Koshland (University of California, Berkeley, USA), M. Singleton (Cancer Research UK), F. Solomon (MIT, USA), P. K. Sorger (Harvard Medical School, USA) and M. Winey (University of Colorado, USA) for strains or reagents."

Reference 48 was omitted in the Methods section. This reference should be cited as follows: "GST-MPS1 (ref. 48) was expressed in E. coli BL21 (DE3)plysS cells", under the heading 'Recombinant production, in vitro phosphorylation and phospho-mapping of Cnn1'. The original reference 48 has been re-numbered as reference 49 , and the new reference 48 is as follows:

48. Holinger, E. P et al. Budding yeast centrosome duplication requires stabilization of Spc29 via Mps1-mediated phosphorylation. J. Biol. Chem. 284, 12949-12955 (2009).

These corrections have been made in the PDF and HTML versions of the Article.

\section{ERRATUM}

\section{Intracellular lumen extension requires ERM-1-dependent apical membrane expansion and AQP-8-mediated flux}

Liakot A. Khan, Hongjie Zhang, Nessy Abraham, Lei Sun, John T. Fleming, Matthew Buechner, David H. Hall and Verena Gobel

Nat. Cell Biol. 15, 143-156 (2013); published online 20th January 2013; corrected online 11 th February 2013

In the version of this Article that was originally published, the first sentence of the second paragraph under the heading 'An RNAi modifier screen reveals a requirement of AQP-8/aquaporin for the ERM-1[++] excretory canal phenotype' (in the Results section) should have read: "Suppression of $a q p-8(R N A i)$ was confirmed in..." The first sentence in the caption of Fig. 1i-l" should have read: "ERM-1 dose-dependently restricts canal extension." The caption for Fig. 1o should have read: "1/4-extended canal with aligning vacuoles."

These errors have been corrected in the PDF and HTML versions of the Article. 